\title{
Thromboprophylaxis in Neuro Intensive Care Unit
}

\author{
Amlan Swain ${ }^{1}$ Seelora Sahu ${ }^{1}$ Binita Panigrahi² \\ ${ }^{1}$ Department of Anaesthesiology, Tata Main Hospital, Jamshedpur, \\ India \\ 2Department of Critical Care, Tata Main Hospital, Jamshedpur, India
}

\begin{abstract}
Address for correspondence Seelora Sahu, DM (Neuroanaesthesia), Tata Main Hospital, Northern Town, Bistupur, Jamshedpur 831001, India (e-mail: seelora@gmail.com; seelora.sahu@tatasteel.com).
\end{abstract}

J Neuroanaesthesiol Crit Care 2019;6:284-291

\begin{abstract}
Keywords

- thromboprophylaxis

- neurocritical care

- craniotomy

The propensity for venous thromboembolism in patients admitted to neurocritical care setups is high because of predilection toward venous stasis as well as pathological processes favoring embolus formation and dissemination. The application of routine thromboprophylaxis guidelines in the various subsets of neurologically injured patients is limited by the fear of bleeding inside neurologically significant closed locations. The aim of this review is to lay out thromboprophylaxis guidelines in various subsets of patients admitted to neurocritical care setups according to recent evidence base, which is still an evolving process.
\end{abstract}

\section{Introduction}

Among the plethora of factors contributing to morbidity and mortality of neurosurgically injured patients in neurocritical care setups, venous thromboembolism (VTE) remains an innocuous yet significant factor. The fact that the neurosurgical procedures are lengthy, associated with delayed ambulation and prolonged hospital stay, and the concomitant predisposing comorbidities make neurologically injured patients more prone for development of VTE. Without thromboprophylaxis, deep vein thrombosis (DVT) rates have been found to be significantly high and previous studies have also shown increased incidence of an alarmingly high rate of a major killer, that is, pulmonary embolism. ${ }^{1}$

There is irrefutable evidence to suggest that patients admitted to an intensive care setup are prone for VTE irrespective of the systemic subset they belong to. ${ }^{2,3}$ While thromboprophylaxis has shown to reduce thromboembolism rates in both surgical and medical subsets, an interesting fact is that it reduces mortality only among surgical patients., Considering the aforementioned background detailing the propensity of neurologically injured patients for VTE and the proven role of thromboprophylaxis in reducing mortality among surgical patients in particular and VTE in general, application of thromboprophylaxis to neurologically injured patients in neurocritical patients should be a no brainer. However, the level of evidence for thromboprophylaxis in neurologically injured patients is still a work in progress and is majorly confounded by the probability of hemorrhage and its effects on closed spaces such as cranium and spinal canal. There have been various systematic reviews in the last two decades on the topic of efficacy of thromboprophylaxis to include pharmacological methods in addition to the widely prevalent mechanical modalities. ${ }^{6}$ Various subsets of neurological injury are relevant in present day neurocritical care (-Table 1).

The aim of this review is to elucidate evidence-based recommendations for thromboprophylaxis of various subsets of neurologically injured patients, surgical and otherwise, as well as the clinical caveats of such use. These recommendations are majorly drawn from a comprehensive evidence-based guideline published by the neurocritical care society in $2016 .^{7}$

Table 1 Various major subsets of patients in neurocritical care

\begin{tabular}{|l|l|}
\hline Cranial pathologies & Spinal pathologies \\
\hline Postcraniotomy patients & Post spine surgery \\
Patients of aneurysmal sub- & Spinal injury patients \\
arachnoid hemorrhage & Patients with neuromuscular \\
Stroke & diseases \\
Traumatic brain injury & \\
Patients undergoing endo- & \\
vascular procedures & \\
Patients with intracranial & \\
hemorrhage & \\
\hline
\end{tabular}

Copyright (02019 Indian Society of Neuroanaesthesiology and Critical Care

\section{License terms \\ ()(1) $\Theta \circledast$}


Table 2 Risk factors for venous thromboembolism in patients undergoing craniotomy and spine surgery

\begin{tabular}{|l|l|}
\hline High risk & $\begin{array}{l}\text { Inconsistent/additional risk } \\
\text { factors }\end{array}$ \\
\hline Malignancy & Previous thromboembolism \\
Poor mobility & Renal compromise \\
Prolonged procedure & Obesity \\
Complicated surgery involv- & Cervical and thoracolumbar \\
ing more than one level & surgery \\
Advanced age & Open surgical technique \\
& Poor compliance to throm- \\
& boprophylaxis protocols \\
\hline
\end{tabular}

\section{Postcraniotomy Patients}

Neurosurgical patients undergoing craniotomy have demonstrated a clinically significant increased risk of DVT, pulmonary embolism (PE), and VTE, especially in the setting of perioperative neurocritical care which plays an integral part in the hospital course of majority of such patients. Various reasons such as concomitant malignancy, long procedural duration, and release of procoagulant factors have been established while some risk factors have proven inconsistent. 8-10 $^{-1}$ The risk factors associated with increased risk of DVT is the same as those undergoing spine surgeries and are set out in - Table 2. The incidence of DVT and PE with or without thromboprophylaxis in patients undergoing craniotomy has shown marked variation in various study populations. ${ }^{8,11-13}$ The American College of Surgeons - National Surgical Quality Improvement Program (ACS-NSQIP) data from 2006 to 2012 for VTE in craniotomy patients vary from 1.7 to $3.2 \%$ with PE rates of $1.4 \%$ and DVT rates of $2.6 \%{ }^{8,11,12}$ Recent studies have shown higher rates of VTE, especially in patients undergoing neurosurgical removal of brain tumors with rates of DVT as high as $14 \%$. $1,9,10,14-16$

There have been several studies examining the virtues of implementing chemical DVT prophylaxis in patients undergoing craniotomy. ${ }^{17-20}$ Moreover, there have been several meta-analyses that have attempted choice of techniques and the commensurate level of evidence of such maneuvers. ${ }^{21-23}$ However, the drawbacks of many of the trials included in the meta-analyses is that they are old and do not include mechanical thromboprophylaxis, which is a matter of routine these days.

Intermittent pneumatic compression (IPC) stockings are a form of mechanical thromboprophylaxis, which obviates the risk and harmful effects of bleeding inside a closed skull cavity. ${ }^{24}$ IPC has shown significant effect in decreasing incidence of DVT and related complications in patients undergoing neurosurgical procedures in the brain. ${ }^{25}$ However, the fact that these devices are not very well tolerated by patients and are cumbersome to use by critical care staff means that they are yet to achieve their full potential. ${ }^{26,27}$ A study in China among the staff in the intensive care unit (ICU) showed that while only half of them practiced mechanical thromboprophylaxis, a quarter of people working in the ICUs (nearly $25 \%$ ) had never heard of the same.

Present-day evidence suggests that whenever IPC is used in patients undergoing craniotomy, their use should be commenced in the pre-surgery phase as soon as the patient is admitted to the hospital and continued in the postoperative intensive care setups with frequent monitoring to improve compliance. , $^{128-31}$

The theoretical risk of intracranial bleeding not withstanding pharmacological thromboprophylaxis with low molecular weight heparin (LMWH) or low-dose heparin (LDH) has proven effective both in conjunction with mechanical methods and standalone therapy in patients undergoing neurosurgical procedures and craniotomies. ${ }^{21-23}$ There is ample evidence to suggest that pharmacological thromboprophylaxis with LMWH and LDH is not associated with increased bleeding risk in patients undergoing craniotomies, especially when such therapy is started after the first postoperative day when surgical hemostasis has been more or less achieved. ${ }^{16,32-34}$

\section{Thromboprophylaxis recommendation in craniotomy (strong recommendation, moderate quality of evidence) \\ - Mechanical thromboprophylaxis ${ }^{1,7}$}

- IPC or gradient compression stockings should be used before surgery and following admission in elective and emergency craniotomies, respectively.

- Pharmacological thromboprophylaxis (LMWH/LDH). ${ }^{1,7}$

- Should be ideally deferred till 24 hours after surgery.

- Preferably used in high-risk population after radiologically confirming hemostasis.

- Duration of thromboprophylaxis till discharge. ${ }^{1}$

\section{Thromboprophylaxis in Patients Undergoing Spine Surgery}

The incidence of DVT and VTE is radically less in patients undergoing surgery on the spine vis-a-vis patients in whom surgery is done on the brain. The DVT rates in this aforementioned subgroup of neurosurgical patients is $0.7 \%$ and thromboembolic complications is around 0.4\%. ${ }^{11,35-37}$ Another interesting fact noted in these patients is that almost $50 \%$ of these patients manifest the signs and symptoms of DVT after they are discharged from the hospital. ${ }^{38}$

Just like patients undergoing craniotomy, certain patients have demonstrated higher risk of thromboembolic complications in patients undergoing spinal procedures. $.^{35,37,39-41} \mathrm{How}-$ ever, the predictive models based on these risk factors are yet tbe validated.

Systematic reviews of effect of thromboprophylaxis on patients undergoing spinal surgeries have revealed rates of DVT in the range of 2.7 to $5.8 \%$ in patients not receiving thromboprophylaxis. Patients with mechanical thromboprophylaxis in spine surgery have revealed DVT rates of around 1.8\% (both GCS and IPC combined). Patients receiving a combination of mechanical and pharmacological thromboprophylaxis fared the best, they had a pooled DVT rate of less than $0.01 \% .40,42,43$ There is a fear of development of a spinal hematoma in patients receiving pharmacological thromboprophylaxis in patients undergoing surgery on the spine. ${ }^{44,45}$ 
The said premise has thankfully been proven to be a theoretical one and even the reported rates of epidural hematoma are highly insignificant $(\sim 0.2 \%) .^{40,42,46-48}$ Bodies of repute such as the North American Spine Society as well as the ACCP guidelines recommend mechanical thromboprophylaxis (IPC preferred) only in patients with additional high-risk factors throughout the perioperative period ( - Table 2 ). In these patients, pharmacological thromboprophylaxis in the form of LMWH can be started in the postoperative period after 24 hours, especially when probability of bleeding is minimal. ${ }^{44,49}$ Like in patients with craniotomy, mechanical thromboprophylaxis should be initiated as soon as possible after hospital admission and steps are desirable to establish protocols which would encourage proper use and compliance to protocol. ${ }^{1}$

\section{Thromboprophylaxis recommendations in spine surgeries}

- Standard elective surgery without additional risk factors, ambulatory surgery (weak recommendation, low quality of evidence $)^{1,7}$
- early mobilization
- no active thromboprophylaxis /IPC only

- Standard elective surgery with risk factors, complicated spinal surgery (strong recommendation, moderate quality of evidence $)^{1,7}$

- IPC preoperatively

- Add LMWH/UFH in postoperative phase when risk of bleeding is low (24 hours when hemostasis occurs)

- IVC filter (weak recommendation, low quality of evidence) ${ }^{7}$

- Not to be used routinely

- Temporary measure only in patients with DVT with contraindication to anticoagulants or in patients with proven DVT and PE

\section{Thromboprophylaxis in spinal cord injury}

- Start within 72 hours of injury (strong recommendation, high quality of evidence $)^{7}$

- If bleeding risk is low or hemostasis is achieved-LMWH/ UFH (Strong recommendation, moderate quality of evidence $)^{7}$

- If bleeding risk is high (patient on chronic anticoagulant and antiplatlet therapy, patients with coagulopathies, severe liver disease $)^{7}$

- IPC (weak recommendation, low quality of evidence)

- Continue till discharge

\section{Thromboprophylaxis in Aneurysmal Subarachnoid Hemorrhage}

Incidence of DVT ranges from 1.5 to $2.4 \%$ while the incidence of PE ranges from 1.2 to $2 \%$ in patients with aneurysmal subarachnoid hemorrhage (SAH) ${ }^{50-52}$ Evidence suggests that use of LMWH is associated with increased risk of intracranial bleeding in patients with cerebral aneurysms undergoing surgical occlusion. ${ }^{53,54}$ Mechanical methods seem to be more efficacious and safe in patients with aneurysmal SAH. ${ }^{55}$ Present recommendations advocate use of either or combination of pneumatic compression device and compression stockings before occlusion of aneurysm (level class II B). ${ }^{29,56}$ This form of mechanical thromboprophylaxis normally suffices for patients with low risk of DVT in postoperative periods. However, in patients with high risk of DVT, LMWH therapy can be started after 12 hours of surgical occlusion and immediately after endovascular coiling (level class II B). ${ }^{56}$

\section{Thromboprophylaxis recommendation in intracranial aneurysm}

- In all patients with aneurysmal SAH other than ruptured aneurysm, for surgery-UFH (better than LDH) (strong recommendation, low quality of evidence $)^{1,7}$

- Use of mechanical thromboprophylaxis-IPC as soon as patient is admitted to the hospital (strong recommendation, moderate quality of evidence)

- Patients undergoing clipping or coiling of ruptured aneurysm-UFH (weak recommendation, low quality of evidence) $)^{1,7}$

- 24 hours after clipping

- Immediately after coiling

\section{Patients Undergoing Neuroendovascular Procedures}

There has been an advent of neurointerventional endovascular techniques such as coil embolization of intracranial aneurysms as well as the proliferation of adjunctive techniques such as balloon and stent-assisted techniques. ${ }^{57}$ Such patients exhibit high propensity for thromboembolic complications with reported periprocedural stroke in the range of 2.3 to $10.4 \% .^{57}$ The postulated mechanisms for a greater tendency toward thromboembolic complications in this patient subgroup include trauma to the cranial vascular tree with resultant thrombosis, migration of coil, and embolization and dislodgement of thrombus in the aneurysm. ${ }^{58}$

Periprocedural heparin administration is especially being favored in patients undergoing coiling for unruptured aneurysms. ${ }^{58,59}$ In patients with ruptured aneurysms, bleeding risks outweigh the potential benefits. Antiplatelet agents (clopidogrel and aspirin) have also been explored in subjects undergoing coiling- and device-assisted procedures to treat cerebral aneurysms. ${ }^{60,61}$ The general consensus for the use of these agents is to employ dual antiplatelet therapy (aspirin $100 \mathrm{mg}$ per day and clopidogrel $75 \mathrm{mg}$ per day), especially in patients with challenging aneurysm anatomy necessitating the use of multiple catheters and stent assistance during the procedure. ${ }^{62,63}$ There has been conflicting evidence for the potential of bleeding complications due to antiplatelet therapy in patients presenting with ruptured aneurysms. ${ }^{63,64}$

Present-day recommendations for patients undergoing endovascular coiling of cerebral aneurysms are elucidated below: 
Thromboprophylaxis recommendation in neuroendovascular procedures (weak recommendation, low quality of evidence)

- Patients without hemiparesis (elective procedure) (weak recommendation, very low quality of evidence $)^{7}$

- Early ambulation

- Mechanical thromboprophylaxis-nneed of UFH/LDH

- Patients with hemiparesis (stroke/other neurological insult) (weak recommendation, low quality of evidence) ${ }^{7}$

- UFH with/without mechanical thromboprophylaxiswithin 24 hours (measure ATT)

- Elective intracranial/intra-arterial procedures (weak recommendation, low quality of evidence $)^{7}$

- IPC or GCS until patient ambulates plus LMWH/UFH

- Coiling of unruptured aneurysm ${ }^{62,63}$

- Dual antiplatelet therapy to be started 4 days before procedure

- After placement of sheath, administer intravenous heparin with target ACT of 250 to 300 seconds.

- Coiling of ruptured aneurysm ${ }^{62,63}$

- Withhold dual antiplatelet therapy (DAT) before the procedure, can be considered in the postoperative period.

- Heparin therapy (femoral)

- Half dose after sheath insertion

- Remaining dose after coil placement

\section{Thromboprophylaxis in Stroke}

Incidence of DVT in patients with stroke is high and variable (10-75\%). ${ }^{65,66}$ Clinical manifestations of DVT appears in 2 to $10 \%$ of patients in acute stroke with peak onset between days 2 and 7. ${ }^{67-69}$ The importance of thromboprophylaxis in patients with stroke can be gauged by the fact that PE accounts for as much as 13 to $25 \%$ early deaths in stroke and is overall the most common cause of mortality in stroke. ${ }^{67}$ Known risk factors for DVT in acute stroke consists of old age, hemiparesis, lack of mobility, a high National Institutes of Health Stroke Scale (NIHSS) score, female sex, and patients of intra-arterial tissue plasminogen activator. ${ }^{67}$

Several trials, such as CLOTS, PREVAIL, and the International Stroke Trial, as well as several comprehensive reviews have attempted to script guidelines for use of mechanical and pharmacological thromboprophylaxis in patients suffering stroke during the acute as well as the rehabilitation phase. ${ }^{67,68,70-75}$ While considering pharmacological thromboprophylaxis it is worthwhile to note that while aspirin has been shown to be ineffective as a single therapy, newer agents such as anti Xa danaproid and rosuvastatin have shown promise. ${ }^{76-78}$

Various international bodies have come up with guidelines for thromboprophylaxis in patients with acute stroke. They are summarized below along with the relevant level of evidence. ${ }^{67}$

\section{Thromboprophylaxis recommendations in ischemic stroke}

- Early mobilization and good hydration (strong recommendation, low quality of evidence) $)^{7,67}$

- All patients with stroke

- Stroke patients with restricted or no mobility (strong recommendation, high quality of evidence $)^{7,67}$

- Pharmacological prophylaxis-LMWH/LDH started immediately (unless contraindication to anticoagulants) ${ }^{7,67}$ - LMWH preferred to $\mathrm{UFH}^{7,67}$

- In patients with renal failure-prefer LDH-grade $B^{67}$

- Pharmacologic prophylaxis should be started within 48 hours of stroke and continued till 2 weeks ${ }^{67}$

- Watch for hemorrhagic transformation in CT scanindication to stop anticoagulation-grade $\mathrm{D}^{67}$

- Aspirin ${ }^{67}$

- Can be used concomitant to other pharmacological thromboprophylaxis

- Should not be used as sole therapy in Stroke patients undergoing decompressive proceure ${ }^{7}$

- UFH/LMWH with/without IPC-immediate postsurgery or postprocedure state-except when patient has received rTPA

- If rTPA started-delay pharmacological methods for 24 hours

\section{Mechanical thromboprophylaxis in patients with stroke} (strong recommendation, moderate quality of evidence) ${ }^{67}$

- Should not be used as standalone therapy in patients with stroke (grade B)

- Can be used as standalone therapy in patients with contraindication to pharmacological methods (grade B)

- In such patients (contraindication to pharmacological thromboprophylaxis) IPC along with GCS is preferred

\section{Thromboprophylaxis in Traumatic Brain Injury}

Incidence of DVT in traumatic brain injury (TBI) could be as high as $54 \%{ }^{79}$ The Brain Trauma Foundation has suggested that LMWH along with mechanical thromboprophylaxis be used in TBI to prevent DVT. ${ }^{80}$

The primary concern in a TBI is the expansion of the intracranial hematoma. ${ }^{81}$ Phelan et al have found that mostly all low-risk ICH patients experience a spontaneous expansion of the hematoma at 48 hours of injury, and most patients with moderate- to high-risk ICH experienced an expansion at 72 hours. ${ }^{82}$ Thus, it would seem logical that thromboprophylaxis should start after 48 hours of lowrisk and 72 hours of moderate- to high-risk hemorrhages. What confounds matters is the fact that thromboprophylaxis during the 72 hours post injury was associated with hematoma expansion in patients with a pre-existing $\mathrm{ICH}^{83}$ It has also been shown that ICH expansion was more when 
chemoprophylaxis was done with unfractionated heparin, than $\mathrm{LMWH}$, making the latter the preferred agent. ${ }^{83}$ It is pertinent to note that spontaneous expansion of hematoma may occur, which is not related to DVT prophylaxis with drugs. ${ }^{84}$

\section{Thromboprophylaxis recommendations in patients with TBI (weak recommendation, low quality of evidence)}

- LMWH/LDH to be used in combination with mechanical thromboprophylaxis-watch for signs and symptoms of expansion in hemorrhage ${ }^{1,7}$

- Pharmacological thromboprophylaxis to be used if brain injury is stable and benefits of such treatment outweigh the risk of intracranial hemorrhage $e^{1,7}$

- IPC-within 24 hours of TBI or within 24 hours after craniotomy ${ }^{1,7}$

- LMWH/UFH-24 t48 hours after TBI or 24 hours after craniotomy ${ }^{1,7}$

\section{Thromboprophylaxis in ICH}

Prevalence of symptomatic DVT in patients with ICH is estimated to be 1 to $5 \%$ with PE rates of 0.5 to $2 \% .85,86 \mathrm{Sev}$ eral trails have established the effectiveness of mechanical methods. ${ }^{28,75} \mathrm{~A}$ comprehensive meta-analysis has established advantages of pharmacological thromboprophylaxis without significant effects on hematoma expansion and outcome in patients with $\mathrm{ICH}^{87}$

\section{Thromboprophylaxis recommendations in patients with ICH}

- Mechanical thromboprophylaxis (IPC and or GCS)-start at hospital admission (strong recommendation, high quality of evidence $)^{1,7}$

- Stable hematoma or normal coagulation statusprophylactic UFH/LMWH (weak recommendations, low quality of evidence) $)^{1,7}$

\section{Thromboprophylaxis in Neuromuscular Diseases}

Immobility, prolonged hospitalization in critical care, and respiratory failure are the reasons for high propensity of these patients to develop DVT and its complications $($ rates of DVT $=4-7 \%$, risk of PE $=3-7 \%$ ). 8 -91 Available recommendations in this subgroup are basically an extrapolation of evidence of critically ill patients and patients with spinal cord injury and are enumerated as follows:

\section{Thromboprophylaxis recommendations in patients with neuromuscular disorders7}

- Pharmacological prophylaxis(UFH/LMWH/Fondaparinux) preferred (strong recommendation, moderate quality of evidence $)^{7}$
- Bleeding risk high (patients on anticoagulants, coagulopathies, liver disease, etc.)

- IPC preferred (strong recommendation, moderate quality of evidence) $)^{7}$

- Combination of pharmacological and mechanical thromboprophylaxis, whenever possible (weak recommendation, low quality of evidence $)^{7}$

- Duration of VTE prophylaxis ${ }^{7}$

- Minimum for duration of hospitalization during acute phase

- Patient is able to ambulate

\section{Conclusion}

With burgeoning evidence in neurologically injured patients it is becoming increasingly clear that thromboprophylaxis has got a significant role in prevention of VTE and the attendant complications and has a potential to improve outcome in various neurocritical patient subgroups. The fear of hemorrhage notwithstanding, especially in high-risk population, the beneficial effects are far more remarkable. There are still areas where focused research would be required, especially to facilitate guideline synthesis in specific patient subgroups.

\section{Funding}

This review received the department and institutional support.

\section{Conflict of Interest}

None declared.

\section{References}

1 Faraoni D, Comes RF, Geerts W, Wiles MD; ESA VTE Guidelines Task Force. European guidelines on perioperative venous thromboembolism prophylaxis: Neurosurgery. Eur J Anaesthesiol 2018;35(2):90-95

2 Castellucci LA, Wells PS, Duffett L. Nonleg venous thrombosis in critically ill adults. JAMA 2015;313(4):411-412

3 Lim W, Meade M, Lauzier F, et al. Prophylaxis for Thrombormbolism in Critical Care Trial Investigators. Failure of anticoagulant thromboprophylaxis: risk factors in medical-surgical critically ill patients. Crit Care Med 2015;43(2):401-410

4 Ejaz A, Ahmed MM, Tasleem A, et al. Thromboprophylaxis in intensive care unit patients: a literature review. Cureus 2018;10(9):e3341

5 Hull RD, Schellong SM, Tapson VF, et al; EXCLAIM (Extended Prophylaxis for Venous ThromboEmbolism in Acutely Ill Medical Patients With Prolonged Immobilization) study. Extended-duration venous thromboembolism prophylaxis in acutely ill medical patients with recently reduced mobility: a randomized trial. Ann Intern Med 2010;153(1):8-18

6 Khan NR, Patel PG, Sharpe JP, Lee SL, Sorenson J. Chemical venous thromboembolism prophylaxis in neurosurgical patients: an updated systematic review and meta-analysis. J Neurosurg 2018;129(4):906-915

7 Nyquist P, Bautista C, Jichici D, et al. Prophylaxis of venous thrombosis in neurocritical care patients: an evidence-based guideline: a statement for healthcare professionals from the Neurocritical Care Society. Neurocrit Care 2016;24(1):47-60 
8 Kimmell KT, Jahromi BS. Clinical factors associated with venous thromboembolism risk in patients undergoing craniotomy. J Neurosurg 2015;122(5):1004-1011

9 Chaichana KL, Pendleton C, Jackson C, et al. Deep venous thrombosis and pulmonary embolisms in adult patients undergoing craniotomy for brain tumors. Neurol Res 2013;35(2):206-211

10 Smith TR, Nanney AD II, Lall RR, et al. Development of venous thromboembolism (VTE) in patients undergoing surgery for brain tumors: results from a single center over a 10 year period. J Clin Neurosci 2015;22(3):519-525

11 Rolston JD, Han SJ, Bloch O, Parsa AT. What clinical factors predict the incidence of deep venous thrombosis and pulmonary embolism in neurosurgical patients? J Neurosurg 2014;121(4):908-918

12 Algattas H, Kimmell KT, Vates GE, Jahromi BS. Analysis of venous thromboembolism risk in patients undergoing craniotomy. World Neurosurg 2015;84(5):1372-1379

13 Henwood PC, Kennedy TM, Thomson L, et al. The incidence of deep vein thrombosis detected by routine surveillance ultrasound in neurosurgery patients receiving dual modality prophylaxis. J Thromb Thrombolysis 2011;32(2):209-214

14 Hoefnagel D, Kwee LE, van Putten EHPP, Kros JM, Dirven CM, Dammers R. The incidence of postoperative thromboembolic complications following surgical resection of intracranial meningioma. A retrospective study of a large single center patient cohort. Clin Neurol Neurosurg 2014;123:150-154

15 Qian C, Yan H, Hu X, Zhang W, Liu H. Increased risk of venous thromboembolism in patients with brain tumors: a systematic review and meta-analysis. Thromb Res 2016;137:58-63

16 Streiff MB, Ye X, Kickler TS, et al. A prospective multicenter study of venous thromboembolism in patients with newly-diagnosed high-grade glioma: hazard rate and risk factors. J Neurooncol 2015;124(2):299-305

17 Agnelli G, Piovella F, Buoncristiani P, et al. Enoxaparin plus compression stockings compared with compression stockings alone in the prevention of venous thromboembolism after elective neurosurgery. N Engl J Med 1998;339(2):80-85

18 Cerrat D, Arian C, Fiacchin F. Deep vein thrombosis and low-dose heparin prophylaxis in neurosurgical patients. J Neurosurg 1978;49(3):378-381

19 Melon E, Keravel Y, Gaston A, et al. Deep venous thrombosis prophylaxis by low molecular weight heparin in neurosurgical patients. Anesthesiology 1991;75:A214 (Abstract)

20 Nurmohamed MT, van Riel AM, Henkens CM, et al. Low molecular weight heparin and compression stockings in the prevention of venous thromboembolism in neurosurgery. Thromb Haemost 1996;75(2):233-238

21 Collen JF, Jackson JL, Shorr AF, Moores LK. Prevention of venous thromboembolism in neurosurgery: a metaanalysis. Chest 2008;134(2):237-249

22 Hamilton MG, Yee WH, Hull RD, Ghali WA. Venous thromboembolism prophylaxis in patients undergoing cranial neurosurgery: a systematic review and meta-analysis. Neurosurgery 2011;68(3):571-581

23 Salmaggi A, Simonetti G, Trevisan E, et al. Perioperative thromboprophylaxis in patients with craniotomy for brain tumours: a systematic review. J Neurooncol 2013;113(2):293-303

24 Geerts WH, Bergqvist D, Pine GF, et al. Prevention of venous thromboembolism:. American College of Chest Physicians Evidence-Based Clinical Practice Guidelines (8th Edition) Chest 2008;133(6, Suppl):381S-453S

25 Frisius J, Ebeling M, Karst M, et al. Prevention of venous thromboembolic complications with and without intermittent pneumatic compression in neurosurgical cranial procedures using intraoperative magnetic resonance imaging. A retrospective analysis. Clin Neurol Neurosurg 2015;133:46-54

26 Sobieraj-Teague M, Hirsh J, Yip G, et al. Randomized controlled trial of a new portable calf compression device (Venowave) for prevention of venous thrombosis in high-risk neurosurgical patients. J Thromb Haemost 2012;10(2):229-235

27 Lacut K, Bressollette L, Le Gal G, et al. VICTORIAh (Venous Intermittent Compression and Thrombosis Occurrence Related tIntra-cerebral Acute hemorrhage) Investigators. Prevention of venous thrombosis in patients with acute intracerebral hemorrhage. Neurology 2005;65(6):865-869

28 Morgenstern LB, Hemphill JC. Anderson C, III, American Heart Association Stroke Council and Council on Cardiovascular Nursing, et al. Guidelines for the management of spontaneous intracerebral hemorrhage: a guideline for healthcare professionals from the American Heart Association/American Stroke Association. Stroke 2010;41(9):2108-2129

29 Diringer MN, Bleck TP, Claude Hemphill J, III, Neurocritical Care Society, et al. Critical care management of patients following aneurysmal subarachnoid hemorrhage: recommendations from the Neurocritical Care Society's Multidisciplinary Consensus Conference. Neurocrit Care 2011;15(2):211-240

30 Steiner T, Al-Shahi Salman R, Beer R, et al. European Stroke Organisation. European Stroke Organisation (ESO) guidelines for the management of spontaneous intracerebral hemorrhage. Int J Stroke 2014;9(7):840-855

31 Hemphill JC. Greenberg SM, Anderson CS, III, American Heart Association Stroke Council, et al. Council on Cardiovascular and Stroke Nursing Council on Clinical Cardiology. Guidelines for the management of spontaneous intracerebral hemorrhage: a guideline for healthcare professionals from the American Heart Association/American Stroke Association. Stroke 2015;46(7):2032-2060

32 Chibbar S, Tacconi L. Safety of deep venous thrombosis prophylaxis with low-molecular-weight heparin in brain surgery. Prospective study on 746 patients. Surg Neurol 2008;70(2):117-121

33 Goldhaber SZ, Dunn K, Gerhard-Herman M. Park JK, Black PM. Low rate of venous thromboembolism after craniotomy for brain tumor using multimodality prophylaxis. Chest 2002;122(6):1933-1937

34 Dickinson LD, Miller LD, Patel CP, Gupta SK. Enoxaparin increases the incidence of postoperative intracranial hemorrhage when initiated preoperatively for deep venous thrombosis prophylaxis in patients with brain tumors. Neurosurgery 1998;43(5):1074-1081

35 Schoenfeld AJ, Herzog JP, Dunn JC. Bader JO, Belmont PJ Jr. Patient-based and surgical characteristics associated with the acute development of deep venous thrombosis and pulmonary embolism after spine surgery. Spine 2013;38(21):1892-1898

36 Gephart MGH, Zygourakis CC, Arrig RT, Kalanithi PS, Lad SP, Boakye M. Venous thromboembolism after thoracic/thoracolumbar spinal fusion. World Neurosurg 2012;78(5):545-552

37 Wang CY, Ignjatovic V, Francis P, et al. Risk factors and clinical features of acute pulmonary embolism in children from the community. Thromb Res 2016;138:86-90

38 Fang MC, Maselli J, Lurie JD, Lindenauer PK, Pekow PS, Auerbach AD. Use and outcomes of venous thromboembolism prophylaxis after spinal fusion surgery. J Thromb Haemost 2011;9(7):1318-1325

39 Yoshioka K, Murakami H, Demura S, Kat S, Tsuchiya H. Prevalence and risk factors for development of venous thromboembolism after degenerative spinal surgery. Spine 2015;40(5):E301

40 Gerlach R, Raabe A, Beck J, Woszczyk A, Seifert V. Postoperative nadroparin administration for prophylaxis of thromboembolic events is not associated with an increased risk of hemorrhage after spinal surgery. Eur Spine J 2004;13(1):9-13

41 Sansone JM, del Rio AM, Anderson PA. The prevalence of and specific risk factors for venous thromboembolic disease following elective spine surgery. J Bone Joint Surg Am 2010;92(2):304-313 
42 Senders ZJ, Zussman BM, Maltenfort MG, Sharan AD, Ratliff JK, Harrop JS. The incidence of pulmonary embolism (PE) after spinal fusions. Clin Neurol Neurosurg 2012;114(7):897-901

43 Glotzbecker MP, Bon CM, Wood KB, Harris MB. Thromboembolic disease in spinal surgery: a systematic review. Spine 2009;34(3):291-303

44 Bono CM, Watters WC II, Heggeness $\mathrm{MH}$, et al. An evidence-based clinical guideline for the use of antithrombotic therapies in spine surgery. Spine J 2009;9(12):1046-1051

45 Glotzbecker MP, Bono CM, Harris MB, Brick G, Heary RF, Wood KB. Surgeon practices regarding postoperative thromboembolic prophylaxis after high-risk spinal surgery. Spine 2008;33(26):2915-2921

46 Cox JB, Weaver KJ, Neal DW, Jacob RP, Hoh DJ. Decreased incidence of venous thromboembolism after spine surgery with early multimodal prophylaxis: Clinical article. J Neurosurg Spine 2014;21(4):677-684

47 Cunningham JE, Swamy G, Thomas KC. Does preoperative DVT chemoprophylaxis in spinal surgery affect the incidence of thromboembolic complications and spinal epidural hematomas? J Spinal Disord Tech 2011;24(4):E31-E34

48 Strom RG, Frempong-Boadu AK. Low-molecular-weight heparin prophylaxis $24 \mathrm{t} 36$ hours after degenerative spine surgery: risk of hemorrhage and venous thromboembolism. Spine 2013;38(23):E1498-E1502

49 Gould MK, Garcia DA, Wren SM, et al. Prevention of VTE in nonorthopedic surgical patients: antithrombotic therapy and prevention of thrombosis, 9th ed: American College of Chest Physicians Evidence-Based Clinical Practice Guidelines. Chest 2012;141:e227S-e277S

50 Serrone JC, Wash EM, Hartings JA, Andaluz N, Zuccarello M. Venous thromboembolism in subarachnoid hemorrhage. World Neurosurg 2013;80(6):859-863

51 Vespa P; Participants in the International Multi-Disciplinary Consensus Conference on the Critical Care Management of Subarachnoid Hemorrhage. Deep venous thrombosis prophylaxis. Neurocrit Care 2011;15(2):295-297

52 Ray WZ, Strom RG, Blackburn SL, Ashley WW, Sicard GA, Rich KM. Incidence of deep venous thrombosis after subarachnoid hemorrhage. J Neurosurg 2009;110(5):1010-1014

53 Siironen J, Juvela S, Varis J, et al. Neffect of enoxaparin on outcome of aneurysmal subarachnoid hemorrhage: a randomized, double-blind, placebo-controlled clinical trial. J Neurosurg 2003;99(6):953-959

54 Juvela S, Siironen J, Varis J, Poussa K, Porras M. Risk factors for ischemic lesions following aneurysmal subarachnoid hemorrhage. J Neurosurg 2005;102(2):194-201

55 Black PM, Baker MF, Snook CP. Experience with external pneumatic calf compression in neurology and neurosurgery. Neurosurgery 1986;18(4):440-444

56 Steiner T, Juvela S, Unterberg A, Jung C, Forsting M, Rinkel G; European Stroke Organization. European Stroke Organization guidelines for the management of intracranial aneurysms and subarachnoid haemorrhage. Cerebrovasc Dis 2013;35(2):93-112

57 Ishibashi T, Murayama Y, Saguchi T, et al. Thromboembolic events during endovascular coil embolization of cerebral aneurysms. Interv Neuroradiol 2006;12(Suppl 1):112-116

58 Qureshi AI, Luft AR, Sharma M, Guterman LR, Hopkins LN. Prevention and treatment of thromboembolic and ischemic complications associated with endovascular procedures: part II-clinical aspects and recommendations. Neurosurgery 2000;46(6):1360-1375, discussion 1375-1376

59 Rahme RJ, Zammar SG, El Ahmadieh TY, El Tecle NE, Ansari SA, Bendok BR. The role of antiplatelet therapy in aneurysm coiling. Neurol Res 2014;36(4):383-388

60 Willard JE, Lange RA, Hillis LD. The use of aspirin in ischemic heart disease. N Engl J Med 1992;327(3):175-181
61 Fiehler J, Ries T. Prevention and treatment of thromboembolism during endovascular aneurysm therapy. Klin Neuroradiol 2009;19(1):73-81

62 Hwang G, Jung C, Park SQ et al. Thromboembolic complications of elective coil embolization of unruptured aneurysms: the effect of oral antiplatelet preparation on periprocedural thromboembolic complication. Neurosurgery 2010;67(3): 743-748

63 Yamada NK, Cross DT, III. Pilgram TK, Moran CJ, Derdeyn CP, Dacey RG, Jr. Effect of antiplatelet therapy on thromboembolic complications of elective coil embolization of cerebral aneurysms. AJNR Am J Neuroradiol 2007;28(9):1778-1782

64 Zhang XD, Wu HT, Zhu J, He ZH, Chai WN, Sun XC. Delayed intracranial hemorrhage associated with antiplatelet therapy in stent-assisted coil embolized cerebral aneurysms. Acta Neurochir Suppl (Wien) 2011;110(Pt 2) :133-139

65 Bembenek J, Karlinski M, Kobayashi A, Czlonkowska A. Early stroke-related deep venous thrombosis: risk factors and influence on outcome. J Thromb Thrombolysis 2011;32(1):96-102

66 Soroceanu A, Burton DC, Oren JH, et al. International Spine Study Group. Medical complications after adult spinal deformity surgery: incidence, risk factors, and clinical impact. Spine 2016;41(22):1718-1723

67 Khan MT, Ikram A, Saeed O, et al. Deep vein thrombosis in acute stroke-a systemic review of the literature. Cureus 2017;9(12):e1982

68 International Stroke Trial Collaborative Group. The International Stroke Trial (IST): a randomised trial of aspirin, subcutaneous heparin, both, or neither among 19435 patients with acute ischaemic stroke. Lancet 1997;349(9065):1569-1581

69 Kelly J, Rudd A, Lewis RR, Coshall C, Moody A, Hunt BJ. Venous thromboembolism after acute ischemic stroke: a prospective study using magnetic resonance direct thrombus imaging. Stroke 2004;35(10):2320-2325

70 Sherman DG, Albers GW, Bladin C, et al. PREVAIL Investigators. The efficacy and safety of enoxaparin versus unfractionated heparin for the prevention of venous thromboembolism after acute ischaemic stroke (PREVAIL Study): an open-label randomised comparison. Lancet 2007;369(9570):1347-1355

71 Bath PM, Iddenden R, Bath FJ. Low-molecular-weight heparins and heparinoids in acute ischemic stroke : a meta-analysis of randomized controlled trials. Stroke 2000;31(7):1770-1778

72 Sandercock PA, Leong TS. Low-molecular-weight heparins or heparinoids versus standard unfractionated heparin for acute ischaemic stroke. Cochrane Database Syst Rev 2017; 4:CD000119

73 Kamphuisen PW, Agnelli G, Sebastianelli M. Prevention of venous thromboembolism after acute ischemic stroke. J Thromb Haemost 2005;3(6):1187-1194

74 CLOTS (Clots in Legs Or sTockings after Stroke) Trial Collaboration. Thigh-length versus below-knee stockings for deep venous thrombosis prophylaxis after stroke: a randomized trial. Ann Intern Med 2010;153:553-562

75 Dennis M, Sandercock P, Reid J, Graham C, Forbes J, Murray G; CLOTS (Clots in Legs Or sTockings after Stroke) Trials Collaboration. Effectiveness of intermittent pneumatic compression in reduction of risk of deep vein thrombosis in patients whhave had a stroke (CLOTS 3): a multicentre randomised controlled trial. Lancet 2013;382(9891):516-524

76 Dumas R, Woitinas F, Kutnowski M, et al. A multicentre, double-blind, randomized study tcompare the safety and efficacy of once-daily ORG 10172 and twice-daily low-dose heparin in preventing deep-vein thrombosis in patients with acute ischaemic stroke. Age Ageing 1994;23(6):512-516

77 Li L, Zhang P, Tian JH, Yang K. Statins for primary prevention of venous thromboembolism. Cochrane Database Syst Rev 2014;12(12):CD008203 
78 Lansberg MG, O’Donnell MJ, Khatri P, et al. Antithrombotic and thrombolytic therapy for ischemic stroke: Antithrombotic Therapy and Prevention of Thrombosis, 9th ed: American College of Chest Physicians Evidence-Based Clinical Practice Guidelines. Chest 2012;141:e601S-e636S

79 Geerts WH, Code KI, Jay RM, Chen E, Szalai JP. A prospective study of venous thromboembolism after major trauma. N Engl J Med 1994;331(24):1601-1606

80 Brain Trauma Foundation; American Association of Neurological Surgeons; Congress of Neurological Surgeons; Joint Section on Neurotrauma and Critical Care, AANS/CNS, Bratton SL, et al. Guidelines for the management of severe traumatic brain injury. V. Deep vein thrombosis prophylaxis. J Neurotrauma. 2007;24(Suppl 1):S32-6. Erratum in:J Neurotrauma. 2008 Mar;25 (3):276-278

81 Phelan HA. Pharmacologic venous thromboembolism prophylaxis after traumatic brain injury: a critical literature review. J Neurotrauma 2012;29(10):1821-1828

82 Phelan HA, Eastman AL, Madden CJ, et al. TBI risk stratification at presentation: a prospective study of the incidence and timing of radiographic worsening in the Parkland Protocol. J Trauma Acute Care Surg 2012;73(2, Suppl 1):S122-S127

83 Abdel-Aziz H, Dunham CM, Malik RJ, Hileman BM. Timing for deep vein thrombosis chemoprophylaxis in traumatic brain injury: an evidence-based review. Crit Care 2015;19:96

84 Bee TK, Magnotti LJ, Croce MA, et al. Necessity of repeat head $\mathrm{CT}$ and ICU monitoring in patients with minimal brain injury. J Trauma 2009;66(4):1015-1018
85 Goldstein JN, Fazen LE, Wendell L, et al. Risk of thromboembolism following acute intracerebral hemorrhage. Neurocrit Care 2009;10(1):28-34

86 Diringer MN, Skolnick BE, Mayer SA, et al. Thromboembolic events with recombinant activated factor VII in spontaneous intracerebral hemorrhage: results from the Factor Seven for Acute Hemorrhagic Stroke (FAST) trial. Stroke 2010;41(1):48-53

87 Paciaroni M, Agnelli G, Venti M, Alberti A, Acciarresi M, Caso V. Efficacy and safety of anticoagulants in the prevention of venous thromboembolism in patients with acute cerebral hemorrhage: a meta-analysis of controlled studies. J Thromb Haemost 2011;9(5):893-898

88 Kahn SR, Lim W, Dunn AS, et al. Prevention of VTE in nonsurgical patients: Antithrombotic therapy and prevention of thrombosis, 9th ed: American College of Chest Physicians evidence-based clinical practice guidelines. Chest 2012;141(2_suppl):e195Se226S

89 Gaber TA, Kirker SG, Jenner JR. Current practice of prophylactic anticoagulation in Guillain-Barré syndrome. Clin Rehabil 2002;16(2):190-193

90 Moore P, James O. Guillain-Barré Syndrome: incidence, management and outcome of major complications. Crit Care Med 1981;9(7):549-555

91 Ropper AH, Kehne SM. Guillain-Barré syndrome: management of respiratory failure. Neurology 1985;35(11):1662-1665 